Bull. Chem. Soc. Ethiop. 2017, 31(2), 351-359.

ISSN 1011-3924

(c) 2017 Chemical Society of Ethiopia and The Authors

Printed in Ethiopia

DOI: http://dx.doi.org/10.4314/bcse.v31i2.17

\title{
MICROWAVE-ASSISTED SYNTHESIS OF SOME NITRO-BENZIMIDAZOLES AND THEIR SALICYL AND ISATIN SCHIFF BASES
}

\author{
Fatih Yılmaz $^{1 *}$, Nesrin Karaali $^{2}$ and Selami Şaşmaz ${ }^{2}$ \\ ${ }^{1}$ Vocational School of Technical Studies, Department of Chemistry and Chemical Processing \\ Technology, Recep Tayyip Erdogan University, 53100, Rize, Turkey \\ ${ }^{2}$ Department of Chemistry, Faculty of Arts and Sciences, Recep Tayyip Erdoğan University, \\ 53100, Rize, Turkey
}

(Received October 18, 2016; revised August 9, 2017)

\begin{abstract}
A series of 5-nitrosubstituted benzimidazole and 6-nitro substituted benzimidazole derivatives were synthesized starting from iminoester hydrochloride and 4-nitro-o-phenylenediamine under microwave irradiation. The structure of newly synthesized compounds was confirmed by IR, ${ }^{1} \mathrm{H}-\mathrm{NMR},{ }^{13} \mathrm{C}-\mathrm{NMR}$, LC-MS spectroscopy and elemental analysis.
\end{abstract}

KEY WORDS: Benzimidazole, Isatin, Salicyl aldehyde, Iminoester hydrochloride, Microwave irradiation

\section{INTRODUCTION}

Benzimidazole is a bicyclic heterocyclic system consisting of two nitrogen and fused phenyl ring and it shows wide range of biological activities. Therefore, its derivatives are in interest of medicinal chemistry because of their biological activities and clinical applications and they are remarkably effective compounds both with respect to their inhibitory activities and biological activities like including antimicrobial, antifungal, antiviral, antiinflammatory, anticonvulsant, antidepressant, antihypertensive, analgesic, enzyme inhibition and hypoglycemic [1-15].

Schiff bases are aldehyde- or ketone-like compounds in which the carbonyl group is replaced by an imine or azomethine group. They are widely used for industrial purposes and also exhibit a broad range of biological activities. They have been reported in their biological properties, such as, antibacterial, antifungal activities [16-19]. Their metal complexes have been widely studied because they have anticancer and herbicidal applications [20,21]. They serve as models for biologically important species. For example, an imine linkage between the aldehyde derived from vitamin A and the protein opsin in the retina of the eye plays an important role in the chemistry of vision.

Salicyl group is an important group, which shows a positive effect on increasing biological activities of compounds. Because of this biological effect, this group has been used as a side group in design of new biological active compounds. In our previous research on this group, we have seen that this group played a positive role on anticonvulsant activity of triazol-3-on compounds [22].

Isatin is an endogenous compound identified in humans that possesses a wide range of biological activities. Isatin has anxiogenic, anticonvulsant activities and acts as a potent antagonist on a trial natriuretic peptide receptors in vitro [23]. Recently, a number of researchers have been studying the use of isatin in the fight against phytopathogens and as potential herbicides $[24,25]$.

In the design of new bioactive compounds, the synthesis of hybrid molecules containing different pharmacophore groups is a useful strategy [26-42]. In the present study, we have

*Corresponding author. E-mail: fyilmaz@erdogan.edu.tr

This work is licensed under the Creative Commons Attribution 4.0 International License 
synthesized some 5(6)-nitro benzimidazoles and their salicyl and isatin Schiff bases, which are important pharmacophores in drug design, by using microwave irradiation.

\section{EXPERIMENTAL}

All the chemicals were supplied from Merck, Aldrich and Alfa Aesar. Melting points were determined on capillary tubes on Buchi oil heating melting point apparatus and uncorrected. ${ }^{1} \mathrm{H}$ NMR and ${ }^{13} \mathrm{C}$ NMR spectra were performed on Varian-Mercury $400 \mathrm{MHz}$ spectrometer in DMSO- $d_{6}$. The elemental compositions were determined on a Carlo Erba 1106 CHN analyser; the experimental values were in agreement $( \pm 0.4 \%)$ with calculated ones.All reactions were monitored by TLC using precoated aluminum sheets (silica gel $60 \mathrm{~F} 2.540 .2 \mathrm{~mm}$ thickness). A mono mode CEM-Discover Microwave was used in the standard configuration as delivered, including proprietary software. All experiments were carried out in microwave process vials (30 $\mathrm{mL}$ ) with control of the temperature by infrared detection temperature sensor.

\section{Synthesis of compounds 5 a- $f$}

Conventional method. A mixture of compounds $4 \mathbf{a}, \mathbf{b}(0.01 \mathrm{~mol})$ and corresponding salicyl aldehyde derivative $(0.012 \mathrm{~mol})$ in ethanol $(25 \mathrm{~mL}$, containing $0.5 \mathrm{~mL}$ of acetic acid) was refluxed for 6 hours. After completion of the reaction (monitored by TLC, ethyl acetate/hexane, $4: 1$, the mixture was cooled to room temperature and a solid was apperared. This product was filtrated off, dried and recrystallized from ethanol to obtain the pure product.

Microwave method. A mixture of compound 4a, b $(0.01 \mathrm{~mol})$ and corresponding salicyl aldehyde derivative $(0.012 \mathrm{~mol})$ was prepared in ethanol $(10 \mathrm{~mL}$, containing $0.5 \mathrm{~mL}$ of acetic acid) in a microwave process vial $(30 \mathrm{~mL})$. Then, the mixture was subjected to microwave irradiation at $120{ }^{\circ} \mathrm{C}$ for $8 \mathrm{~min}$. After completion of the reaction (monitored by TLC, ethyl acetate/hexane, $4: 1$ ), above purification procedures were applied to obtain the product.

2-[2-(4-Methylbenzyl)-5(6)-nitro-1H-benzimidazol-1-yl]- $N^{\prime}-[(2-h y d r o x y p h e n y l) m e t h y l i d e n e] a c-$ etohydrazide (5a). ${ }^{1} \mathrm{H}$ NMR (400 MHZ, DMSO- $\left.d_{6}\right)(\delta, \mathrm{ppm}): 12.06(\mathrm{~s}, 1 \mathrm{H}, \mathrm{NH}), 10.16(\mathrm{~s}, 1 \mathrm{H}$, $\mathrm{OH}), 8.23-8.04(\mathrm{~s}, 1 \mathrm{H}, \mathrm{CH}, \mathrm{E} / \mathrm{Z}$ geometrical isomer, $\mathrm{E} / \mathrm{Z}$ ratio $72 / 28), 7.80-7.56(\mathrm{~m}, 2 \mathrm{H}, \mathrm{Ar}-\mathrm{H})$, 7.38-7.21 (m, 4H, Ar-H), 7.19-6.89 (m, 5H, Ar-H), 5.47+5.03 (s, $2 \mathrm{H}, \mathrm{NCH}_{2}$, trans and cis amid conformer, cis/trans ratio 73/27), $4.22\left(\mathrm{~s}, 2 \mathrm{H}, \mathrm{CH}_{2}\right), 2.06\left(\mathrm{~s}, 3 \mathrm{H}, \mathrm{CH}_{3}\right) .{ }^{13} \mathrm{C}$ NMR $(400 \mathrm{MHZ}$, DMSO- $\left.d_{6}\right)(\delta, \mathrm{ppm}): 163.7(\mathrm{C}=\mathrm{O}), 153.2\left(\mathrm{C}=\mathrm{N}_{\text {benzimidazole }}\right), 145.3(\mathrm{~N}=\mathrm{CH}), 142.7,140.6,137.6$, 135.8, 131.3, 130.1, 129.9, 128.5, 127.3, 125.6, 124.9, 123.4, 121.6, 118.6, 109.5 (Ar-C), 49.5 $\left(\mathrm{NCH}_{2}\right), 35.1\left(\mathrm{CH}_{2}\right), 20.1\left(\mathrm{CH}_{3}\right)$. Elemental analysis (\% calculated/found) for $\mathrm{C}_{24} \mathrm{H}_{21} \mathrm{~N}_{5} \mathrm{O}_{4}(\mathrm{MW}$ 443.45) C: $65.00 / 64.86$, H: 4.77/4.69, N: 15.79/15.71.

2-[2-(4-Chlorobenzyl)-5(6)-nitro-1H-benzimidazol-1-yl]-N'-[(2-hydroxyphenyl)methylidene]acetohydrazide (5b). ${ }^{1} \mathrm{H}$ NMR (400 MHZ, DMSO- $\left.d_{6}\right)(\delta$, ppm): $11.11(\mathrm{~s}, 1 \mathrm{H}, \mathrm{NH}), 10.16(\mathrm{~s}, 1 \mathrm{H}$, $\mathrm{OH}), 8.31-8.11$ (s, 1H, CH, E/Z geometrical isomer, E/Z ratio 75/25), 7.91-7.53 (m, 2H, Ar-H), 7.38-7.23 (m, 4H, Ar-H), 7.15-6.90 (m, 5H, Ar-H), 5.51+5.10 (s, $2 \mathrm{H}, \mathrm{NCH}_{2}$, trans and cis amid conformer, cis/trans ratio 76/24), $4.20\left(\mathrm{~s}, 2 \mathrm{H}, \mathrm{CH}_{2}\right) .{ }^{13} \mathrm{C}$ NMR (400 MHZ, DMSO- $\left.d_{6}\right)(\delta$, ppm): $165.1(\mathrm{C}=\mathrm{O}), 155.0\left(\mathrm{C}=\mathrm{N}_{\text {benzimidazole }}\right), 146.0(\mathrm{~N}=\mathrm{CH}), 143.1,141.2,139.1,136.0,133.7,131.0$, 129.6, 129.1, 127.0, 125.0, 124.1, 123.0, 122.7, 118.6, 108.1 ( $\mathrm{Ar}-\mathrm{C}), 49.9\left(\mathrm{NCH}_{2}\right), 35.3\left(\mathrm{CH}_{2}\right)$. Elemental analysis (\% calculated/found) for $\mathrm{C}_{23} \mathrm{H}_{18} \mathrm{ClN}_{5} \mathrm{O}_{4}$ (MW 463.87) C: 59.55/59.48, H: 3.91/3.82, N: 15.10/15.02.

2-[2-(4-Methylbenzyl)-5(6)-nitro-1H-benzimidazol-1-yl]-N'-[(5-chloro-2-hydroxyphenyl)methylidene] acetohydrazide (5c). ${ }^{1} \mathrm{H}$ NMR (400 MHZ, DMSO- $\left.d_{6}\right)(\delta, \mathrm{ppm}): 11.13(\mathrm{~s}, 1 \mathrm{H}, \mathrm{NH}), 10.17$ 
$(\mathrm{s}, 1 \mathrm{H}, \mathrm{OH}), 8.36-8.19(\mathrm{~s}, 1 \mathrm{H}, \mathrm{CH}, \mathrm{E} / \mathrm{Z}$ geometrical isomer, $\mathrm{E} / \mathrm{Z}$ ratio 78/22), 7.97-7.63 $(\mathrm{m}, 1 \mathrm{H}$, Ar-H), 7.46-7.39 (m, 4H, Ar-H), 7.28-7.03 (m, 5H, Ar-H), 5.58+5.15 (s, $2 \mathrm{H}, \mathrm{NCH}_{2}$, trans and cis amid conformer, cis/trans ratio 76/24), $4.20\left(\mathrm{~s}, 2 \mathrm{H}, \mathrm{CH}_{2}\right), 2.01\left(\mathrm{~s}, 3 \mathrm{H}, \mathrm{CH}_{3}\right) .{ }^{13} \mathrm{C} \mathrm{NMR}(400$ MHZ, DMSO- $\left.d_{6}\right)(\delta, \mathrm{ppm}): 165.9(\mathrm{C}=\mathrm{O}), 156.8\left(\mathrm{C}=\mathrm{N}_{\text {benzimidazole }}\right), 146.0(\mathrm{~N}=\mathrm{CH}), 143.1,142.1$, $138.9,136.7,136.0,134.2,133.4,132.3,129.0,126.5,124.1,122.1,120.8,120.0,118.6$ (Ar-C), $49.5\left(\mathrm{NCH}_{2}\right), \quad 35.1\left(\mathrm{CH}_{2}\right), 20.1 \quad\left(\mathrm{CH}_{3}\right)$. Elemental analysis $(\%$ calculated/found $)$ for $\mathrm{C}_{24} \mathrm{H}_{20} \mathrm{ClN}_{5} \mathrm{O}_{4}$ (MW 477.90) C: 60.32/60.26, H: 4.22/4.13, N: 14.65/14.57.

2-[2-(4-Chlorobenzyl)-5(6)-nitro-1H-benzimidazol-1-yl]-N'-[(5-chloro-2-hydroxyphenyl)methylidene]acetohydrazide (5d). ${ }^{1} \mathrm{H}$ NMR (400 MHZ, DMSO- $\left.d_{6}\right)(\delta, \mathrm{ppm}): 11.72(\mathrm{~s}, 1 \mathrm{H}, \mathrm{NH}), 10.46$ $(\mathrm{s}, 1 \mathrm{H}, \mathrm{OH}), 8.74-8.11(\mathrm{~s}, 1 \mathrm{H}, \mathrm{CH}, \mathrm{E} / \mathrm{Z}$ geometrical isomer, $\mathrm{E} / \mathrm{Z}$ ratio $75 / 25), 7.99-7.60(\mathrm{~m}, 1 \mathrm{H}$, Ar-H), 7.51-7.40 (m, 3H, Ar-H), 7.32-7.11 (m, 5H, Ar-H), 5.61+5.22 (s, $2 \mathrm{H}, \mathrm{NCH}_{2}$, trans and cis amid conformer, cis/trans ratio 76/24), $4.25\left(\mathrm{~s}, 2 \mathrm{H}, \mathrm{CH}_{2}\right) .{ }^{13} \mathrm{C}$ NMR (400 MHZ, DMSO- $\left.d_{6}\right)$ $(\delta, \mathrm{ppm}): 166.7(\mathrm{C}=\mathrm{O}), 157.3\left(\mathrm{C}=\mathrm{N}_{\text {benzimidazole }}\right), 147.3(\mathrm{~N}=\mathrm{CH}), 145.1,143.6,140.1,138.9,137.0$, 135.2, 134.4, 133.1, 128.0, 125.5, 122.0, 120.1, 119.8, 119.0, $118.3(\mathrm{Ar}-\mathrm{C}), 49.4\left(\mathrm{NCH}_{2}\right), 35.1$ $\left(\mathrm{CH}_{2}\right)$. Elemental analysis (\% calculated/found) for $\mathrm{C}_{24} \mathrm{H}_{20} \mathrm{ClN}_{5} \mathrm{O}_{4}(\mathrm{MW} 498.31) \mathrm{C}: 55.44 / 55.38$, H: 3.44/3.37, N: 14.05/14.01.

2-[2-(4-Methylbenzyl)-5(6)-nitro-1H-benzimidazol-1-yl]-N'-[(5-bromo-2-hydroxyphenyl)methylidene] acetohydrazide (5e). ${ }^{1} \mathrm{H}$ NMR (400 MHZ, DMSO- $\left.d_{6}\right)(\delta, \mathrm{ppm}): 11.76(\mathrm{~s}, 1 \mathrm{H}, \mathrm{NH}), 10.19$ $(\mathrm{s}, 1 \mathrm{H}, \mathrm{OH}), 8.41-8.77(\mathrm{~s}, 1 \mathrm{H}, \mathrm{CH}, \mathrm{E} / \mathrm{Z}$ geometrical isomer, $\mathrm{E} / \mathrm{Z}$ ratio $73 / 27), 7.99-7.81(\mathrm{~m}, 1 \mathrm{H}$, Ar-H), 7.46-7.32 (m, 2H, Ar-H), 7.29-7.07 (m, 6H, Ar-H), 5.60+5.29 (s, $2 \mathrm{H}, \mathrm{NCH}_{2}$, trans and cis amid conformer, cis/trans ratio 79/21), $4.22\left(\mathrm{~s}, 2 \mathrm{H}, \mathrm{CH}_{2}\right), 2.12\left(\mathrm{~s}, 3 \mathrm{H}, \mathrm{CH}_{3}\right) .{ }^{13} \mathrm{C}$ NMR $(400$ MHZ, DMSO- $\left.d_{6}\right)(\delta, \mathrm{ppm}): 168.0(\mathrm{C}=\mathrm{O}), 155.1\left(\mathrm{C}=\mathrm{N}_{\text {benzimidazole }}\right), 149.0(\mathrm{~N}=\mathrm{CH}), 144.1,140.1$, 137.1, 136.7, 135.3, 135.0, 134.4, 133.1, 128.2, 127.5, 126.1, 124.1, 122.8, 120.0, 118.1 (Ar-C), $48.1\left(\mathrm{NCH}_{2}\right), \quad 36.3\left(\mathrm{CH}_{2}\right), 22.3 \quad\left(\mathrm{CH}_{3}\right)$. Elemental analysis (\% calculated/found) for $\mathrm{C}_{24} \mathrm{H}_{20} \mathrm{BrN}_{5} \mathrm{O}_{4}$ (MW 522.35) C: 55.18/55.06, H: 3.86/3.78, N: 13.41/13.34.

2-[2-(4-Chlorobenzyl)-5(6)-nitro-1H-benzimidazol-1-yl]-N'-[(5-bromo-2-hydroxyphenyl)methylidene]acetohydrazide (5f) ${ }^{1} \mathrm{H}$ NMR (400 MHZ, DMSO- $\left.d_{6}\right)(\delta, \mathrm{ppm}): 11.72(\mathrm{~s}, 1 \mathrm{H}, \mathrm{NH}), 10.46$ $(\mathrm{s}, 1 \mathrm{H}, \mathrm{OH}), 8.74-8.11(\mathrm{~s}, 1 \mathrm{H}, \mathrm{CH}, \mathrm{E} / \mathrm{Z}$ geometrical isomer, $\mathrm{E} / \mathrm{Z}$ ratio $75 / 25), 7.99-7.60(\mathrm{~m}, 1 \mathrm{H}$, Ar-H), 7.51-7.40 (m, 3H, Ar-H), 7.32-7.11 (m, 5H, Ar-H), 5.61+5.22 (s, 2H, $\mathrm{NCH}_{2}$, trans and cis amid conformer, cis/trans ratio 76/24), $4.25\left(\mathrm{~s}, 2 \mathrm{H}, \mathrm{CH}_{2}\right) .{ }^{13} \mathrm{C}$ NMR $\left(400 \mathrm{MHZ}, \mathrm{DMSO}-d_{6}\right)$ $(\delta, \mathrm{ppm}): 166.7(\mathrm{C}=\mathrm{O}), 157.3\left(\mathrm{C}=\mathrm{N}_{\text {benzimidazole }}\right), 147.3(\mathrm{~N}=\mathrm{CH}), 145.1,143.6,140.1,138.9,137.0$, 135.2, 134.4, 133.1, 128.0, 125.5, 122.0, 120.1, 119.8, 119.0, 118.3 (Ar-C), $49.4\left(\mathrm{NCH}_{2}\right), 35.1$ $\left(\mathrm{CH}_{2}\right)$. Elemental analysis (\% calculated/found) for $\mathrm{C}_{23} \mathrm{H}_{17} \mathrm{BrClN}_{5} \mathrm{O}_{4}$ (MW 542.77) C: 50.90/50.82, H: 3.16/3.08, N: 12.90/12.82.

\section{Synthesis of compounds $\mathbf{6 a - d}$}

Conventional method. A mixture of compounds $4 \mathbf{a}, \mathbf{b}(0.01 \mathrm{~mol})$ and corresponding isatin derivatives $(0.011 \mathrm{~mol})$ in ethanol $(25 \mathrm{~mL}$, containing $0.5 \mathrm{~mL}$ of acetic acid) was refluxed for 8 hours. After completion of the reaction (monitored by TLC, ethyl acetate/hexane, 5:2), the mixture was cooled to room temperature. The appeared solid was filtrated off, washed with hot ethanol and dried to obtain the pure product.

Microwave method. A mixture ofcompound $4 \mathbf{a}, \mathbf{b}(0.01 \mathrm{~mol})$ and corresponding isatin derivative $(0.011 \mathrm{~mol})$ was prepared in ethanol $(10 \mathrm{~mL}$, containing $0.5 \mathrm{~mL}$ of acetic acid) in a microwave process vial $(30 \mathrm{~mL})$. Then, the mixture was subjected to microwave irradiation at $130{ }^{\circ} \mathrm{C}$ for 10 min. After completion of the reaction (monitored by TLC, ethyl acetate/hexane, 5:2), above purification procedures were applied to obtain the product. 
2-[2-(4-Methylbenzyl)-5(6)-nitro-1H-benzimidazol-1-yl]-N'-[2-oxo-1,2-dihydro-3H-indol-3-ylidene]acetohydrazide (6a). ${ }^{1} \mathrm{H}$ NMR (400 MHZ, DMSO- $\left.d_{6}\right)(\delta, \mathrm{ppm}): 11.95(\mathrm{~s}, 1 \mathrm{H}, \mathrm{NH}), 11.70$ (s, 1H, NH), 8.02-7.73 (m, 2H, Ar-H), 7.62-7.43 (m, 2H, Ar-H), 7.33-7.16 (m, 3H, Ar-H), 7.06$6.77(\mathrm{~m}, 4 \mathrm{H}, \mathrm{Ar}-\mathrm{H}), 5.55+5.27\left(\mathrm{~s}, 2 \mathrm{H}, \mathrm{NCH}_{2}\right.$, trans and cis amid conformer, cis/trans ratio 79/21), 4.16 (s, 2H, $\left.\mathrm{CH}_{2}\right), 2.03\left(\mathrm{~s}, 3 \mathrm{H}, \mathrm{CH}_{3}\right) .{ }^{13} \mathrm{C}$ NMR (400 MHZ, DMSO- $\left.d_{6}\right)(\delta, \mathrm{ppm}): 167.2$ $(\mathrm{C}=\mathrm{O}), 164.1(\mathrm{C}=\mathrm{O}), 156.7\left(\mathrm{C}=\mathrm{N}_{\text {benzimidazole }}\right), 146.1(\mathrm{~N}=\mathrm{CH}), 144.2,143.5,141.7,139.2,137.6$, $135.5,135.0,131.8,129.0,128.7,127.1,125.3,124.3,122.5,118.1,117.3,116.0$ (Ar-C), 50.1 $\left(\mathrm{NCH}_{2}\right), 33.7\left(\mathrm{CH}_{2}\right), 21.1\left(\mathrm{CH}_{3}\right)$. Elemental analysis (\% calculated/found) for $\mathrm{C}_{25} \mathrm{H}_{20} \mathrm{~N}_{6} \mathrm{O}_{4}(\mathrm{MW}$ 468.46) C: 64.10/64.02, H: 4.30/4.21, N: 17.94/17.82.

2-[2-(4-Chlorobenzyl)-5(6)-nitro-1H-benzimidazol-1-yl]-N'-[2-oxo-1,2-dihydro-3H-indol-3-ylidene]acetohydrazide (6b). ${ }^{1} \mathrm{H}$ NMR (400 MHZ, DMSO- $\left.d_{6}\right)(\delta, \mathrm{ppm}): 11.79(\mathrm{~s}, 1 \mathrm{H}, \mathrm{NH}), 11.18$ (s, 1H, NH), 8.09-7.64 (m, 3H, Ar-H), 7.58-7.39 (m, 3H, Ar-H), 7.33-7.10 (m, 2H, Ar-H), 7.03$6.81(\mathrm{~m}, 3 \mathrm{H}, \mathrm{Ar}-\mathrm{H}), 5.59+5.31\left(\mathrm{~s}, 2 \mathrm{H}, \mathrm{NCH}_{2}\right.$, trans and cis amid conformer, cis/trans ratio 80/20), $4.19\left(\mathrm{~s}, 2 \mathrm{H}, \mathrm{CH}_{2}\right) .{ }^{13} \mathrm{C}$ NMR (400 MHZ, DMSO- $\left.d_{6}\right)(\delta, \mathrm{ppm}): 169.1(\mathrm{C}=\mathrm{O}), 165.0$ $(\mathrm{C}=\mathrm{O}), 158.0\left(\mathrm{C}=\mathrm{N}_{\text {benzimidazole }}\right), 147.3(\mathrm{~N}=\mathrm{CH}), 145.0,144.1,143.2,140.3,138.1,136.4,134.0$, 130.1, 128.6, 128.0, 127.2, 124.9, 124.0, 121.2, 119.6, 118.0, 115.3 (Ar-C), $51.6\left(\mathrm{NCH}_{2}\right), 34.2$ $\left(\mathrm{CH}_{2}\right)$. Elemental analysis (\% calculated/found) for $\mathrm{C}_{24} \mathrm{H}_{14} \mathrm{ClN}_{6} \mathrm{O}_{4}$ (MW 488.88) C: $58.96 / 58.85$, H: 3.50/3.43, N: 17.19/17.08.

2-[2-(4-Methylbenzyl)-5(6)-nitro-1H-benzimidazol-1-yl]-N'-[5-nitro-2-oxo-1,2-dihydro-3H-indol-3-ylidene] acetohydrazide (6c). ${ }^{1} \mathrm{H}$ NMR (400 MHZ, DMSO- $\left.d_{6}\right)(\delta, \mathrm{ppm}): 12.02(\mathrm{~s}, 1 \mathrm{H}, \mathrm{NH})$, $11.84(\mathrm{~s}, 1 \mathrm{H}, \mathrm{NH}), 8.11-7.91(\mathrm{~m}, 3 \mathrm{H}, \mathrm{Ar}-\mathrm{H}), 7.82-7.63(\mathrm{~m}, 2 \mathrm{H}, \mathrm{Ar}-\mathrm{H}), 7.53-7.36(\mathrm{~m}, 2 \mathrm{H}, \mathrm{Ar}-\mathrm{H})$, 7.26-6.87 (m, $3 \mathrm{H}, \mathrm{Ar}-\mathrm{H}), 5.58+5.28\left(\mathrm{~s}, 2 \mathrm{H}, \mathrm{NCH}_{2}\right.$, trans and cis amid conformer, cis/trans ratio $79 / 21), 4.19\left(\mathrm{~s}, 2 \mathrm{H}, \mathrm{CH}_{2}\right), 2.11\left(\mathrm{~s}, 3 \mathrm{H}, \mathrm{CH}_{3}\right) .{ }^{13} \mathrm{C}$ NMR (400 MHZ, DMSO-d $\left.d_{6}\right)(\delta, \mathrm{ppm}): 171.2$ $(\mathrm{C}=\mathrm{O}), 167.1(\mathrm{C}=\mathrm{O}), 159.3\left(\mathrm{C}=\mathrm{N}_{\text {benzimidazole }}\right), 148.1(\mathrm{~N}=\mathrm{CH}), 146.7,145.5,142.2,141.0,138.7$, $136.1,134.5,133.6,130.8,128.9,126.2,125.6,123.5,121.4,119.9,115.3,110.0$ (Ar-C), 52.4 $\left(\mathrm{NCH}_{2}\right), 31.9\left(\mathrm{CH}_{2}\right), 20.6\left(\mathrm{CH}_{3}\right)$. Elemental analysis (\% calculated/found) for $\mathrm{C}_{25} \mathrm{H}_{19} \mathrm{~N}_{7} \mathrm{O}_{6}(\mathrm{MW}$ 513.46) C: 58.48/58.41, H: 3.73/3.62, N: 19.10/18.98.

2-[2-(4-Chlorobenzyl)-5(6)-nitro-1H-benzimidazol-1-yl]-N'-[5-nitro-2-oxo-1,2-dihydro-3H-indol-3-ylidene] acetohydrazide (6d). ${ }^{1} \mathrm{H}$ NMR $\left(400 \mathrm{MHZ}, \mathrm{DMSO}-d_{6}\right)(\delta, \mathrm{ppm}): 12.05(\mathrm{~s}, 1 \mathrm{H}, \mathrm{NH})$, 11.79 (s, 1H, NH), 8.19-7.79 (m, 2H, Ar-H), 7.68-7.41 (m, 3H, Ar-H), 7.35-7.06 (m, 2H, Ar-H), 7.00-6.80 (m, 3H, Ar-H), 5.62+5.40 (s, $2 \mathrm{H}, \mathrm{NCH}_{2}$, trans and cis amid conformer, cis/trans ratio 76/24), $4.21\left(\mathrm{~s}, 2 \mathrm{H}, \mathrm{CH}_{2}\right) .{ }^{13} \mathrm{C}$ NMR (400 MHZ, DMSO- $\left.d_{6}\right)(\delta, \mathrm{ppm}): 172.0(\mathrm{C}=\mathrm{O}), 167.2$ $(\mathrm{C}=\mathrm{O}), 159.3\left(\mathrm{C}=\mathrm{N}_{\text {benzimidazole }}\right), 148.1(\mathrm{~N}=\mathrm{CH}), 146.3,145.2,144.3,142.2,139.3,138.1,136.3$, 133.5, 129.1, 127.9, 126.7, 123.9, 122.1, 120.3, 118.9, 117.3, 114.3 (Ar-C), $51.6\left(\mathrm{NCH}_{2}\right), 34.2$ $\left(\mathrm{CH}_{2}\right)$. Elemental analysis (\% calculated/found) for $\mathrm{C}_{24} \mathrm{H}_{14} \mathrm{ClN}_{6} \mathrm{O}_{4}(\mathrm{MW} \mathrm{533.88)} \mathrm{C}:$ 53.99/53.90, H: 3.02/2.91, N: 18.36/18.27.

\section{RESULTS AND DISCUSSION}

The targeted isatin and salicylschiff bases of 5(6)-nitro benzimidazoles (5a-f and 6a-d) were prepared as shown in Scheme 1. Firstly, 5(6)-nitrobenzimidazoleacetohydrazides (4a,b) were synthesized according to the literature [43]. Secondly, various salicyl aldehyde derivatives were reacted with the compound $\mathbf{4 a}, \mathbf{b}$ in ethanol with catalytic amount of glacial acetic acid to obtain the salicyl Schiff bases of 5(6)-nitrobenzimidazoles (5a-f). The lastly, isatin and 5-nitroisatin, separately, were reacted with compounds $\mathbf{4 a}, \mathbf{b}$ in the same condition with compounds 5a-f (Scheme 1). The synthesis of these target compounds performed under microwave irradiation and by using conventional heating procedure. The results of these two methods were compared (Table 1). 


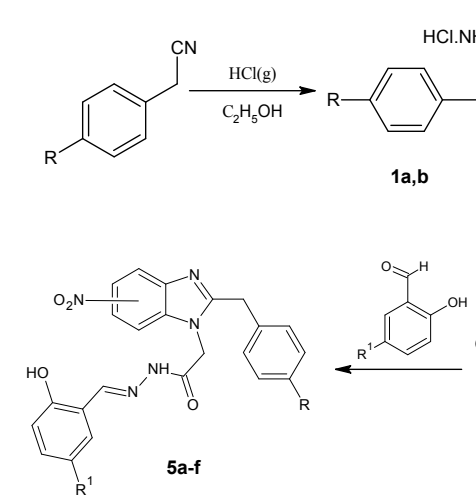<smiles>CC=C(C)OCC</smiles><smiles>O=[N+]([O-])c1ccc2[nH]c(Cc3ccc(I)cc3)nc2c1</smiles>

\begin{tabular}{l|l}
$\mathrm{BrCH}_{2} \mathrm{COOEt}$ & $\mathrm{K}_{2} \mathrm{CO}_{3}$, Acetone
\end{tabular}

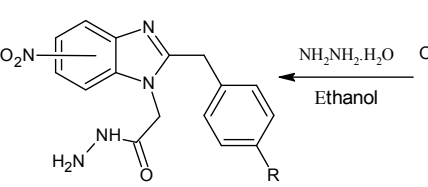

4a,b
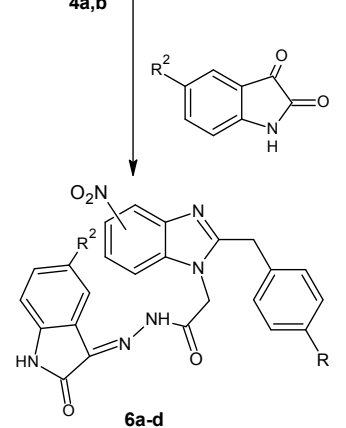

\begin{tabular}{|l|l|l|l|l|l|l|}
\hline & $\mathbf{R}$ & $\mathbf{R}^{1}$ & & $\mathbf{R}$ & $\mathbf{R}^{1}$ & $\mathbf{R}^{2}$ \\
\hline 4a & $\mathrm{CH}_{3}$ & - & $\mathbf{5 e}$ & $\mathrm{CH}_{3}$ & $\mathrm{Br}$ & - \\
\hline 4b & $\mathrm{Cl}$ & - & $\mathbf{5 f}$ & $\mathrm{Cl}$ & $\mathrm{Br}$ & - \\
\hline $\mathbf{5 a}$ & $\mathrm{CH}_{3}$ & $\mathrm{H}$ & $\mathbf{6 a}$ & $\mathrm{CH}_{3}$ & - & $\mathrm{H}$ \\
\hline $\mathbf{5 b}$ & $\mathrm{Cl}$ & $\mathrm{H}$ & $\mathbf{6 b}$ & $\mathrm{Cl}$ & - & $\mathrm{H}$ \\
\hline $\mathbf{5 c}$ & $\mathrm{CH}$ & $\mathrm{Cl}$ & $\mathbf{6 c}$ & $\mathrm{CH}_{3}$ & - & $\mathrm{NO}_{2}$ \\
\hline $\mathbf{5 d}$ & $\mathrm{Cl}$ & $\mathrm{Cl}$ & $\mathbf{6 d}$ & $\mathrm{Cl}$ & - & $\mathrm{NO}_{2}$ \\
\hline
\end{tabular}

Scheme 1. Synthetic route for the benzimidazole derivatives containing salicyl and isatin moieties.

Table 1. Comparison of yield and reaction time of compounds 5a-f and 6a-d.

\begin{tabular}{|c|c|c|c|c|c|}
\hline \multirow{2}{*}{ Compound } & \multirow{2}{*}{$\begin{array}{c}\text { Melting point } \\
\left({ }^{\circ} \mathrm{C}\right)\end{array}$} & \multicolumn{2}{|c|}{ Microwave heating } & \multicolumn{2}{c|}{ Conventional heating } \\
\cline { 3 - 6 } & $247-248$ & Time $(\mathrm{min})$ & Yield (\%) & Time (hour) & Yield (\%) \\
\hline $\mathbf{5 a}$ & $253-254$ & 8 & 86 & 6 & 76 \\
\hline $\mathbf{5 b}$ & $229-230$ & 8 & 78 & 6 & 68 \\
\hline $\mathbf{5 c}$ & $213-214$ & 8 & 82 & 6 & 75 \\
\hline $\mathbf{5 d}$ & $220-221$ & 8 & 84 & 6 & 73 \\
\hline $\mathbf{5 e}$ & $232-233$ & 8 & 81 & 6 & 78 \\
\hline $\mathbf{5 f}$ & $200-201$ & 10 & 93 & 6 & 58 \\
\hline 6a & $216-217$ & 10 & 73 & 8 & 60 \\
\hline 6b & $236-237$ & 10 & 76 & 8 & 51 \\
\hline 6c & $208-209$ & 10 & 71 & 8 & 55 \\
\hline 6d & & & 79 & & \\
\hline
\end{tabular}

Bull. Chem. Soc. Ethiop. 2017, 31(2) 
Spectral investigations of newly synthesized compounds are suitable with the proposed structures. ${ }^{1} \mathrm{H}$ NMR spectra of each compounds showed characteristic signals. $\mathrm{N}=\mathrm{CH}$ and $\mathrm{OH}$, coming from salicyl moiety, signals were shown at about $8.5 \mathrm{ppm}$ and $10.0 \mathrm{ppm}$ in ${ }^{1} \mathrm{H} \mathrm{NMR}$ spectra of compounds 5a-f. $\mathrm{NCH}_{2}$ signals were shown at about 5.0 ppm ${ }^{1} \mathrm{H}$ NMR spectra of compounds 5a-f and 6a-d. NH signal of isatin moiety in compounds 6a-d was shown at about $11.5 \mathrm{ppm}$.<smiles>[R]C=NN([2H])C([R])=O</smiles>

trans, $E$<smiles>[CH]=C</smiles><smiles>[R]C=NN([2H])C([R])=O</smiles>

cis, $E$<smiles>[R]C=NN([TlH])C([R])=O</smiles>

trans, $Z$<smiles>[CH]=C</smiles>

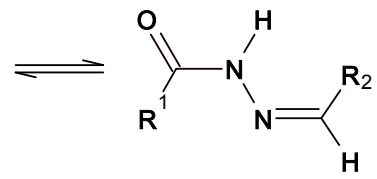

cis, $Z$

Scheme 2. E/Z geometrical isomer and cis/trans amid conformer of compounds 5a-g

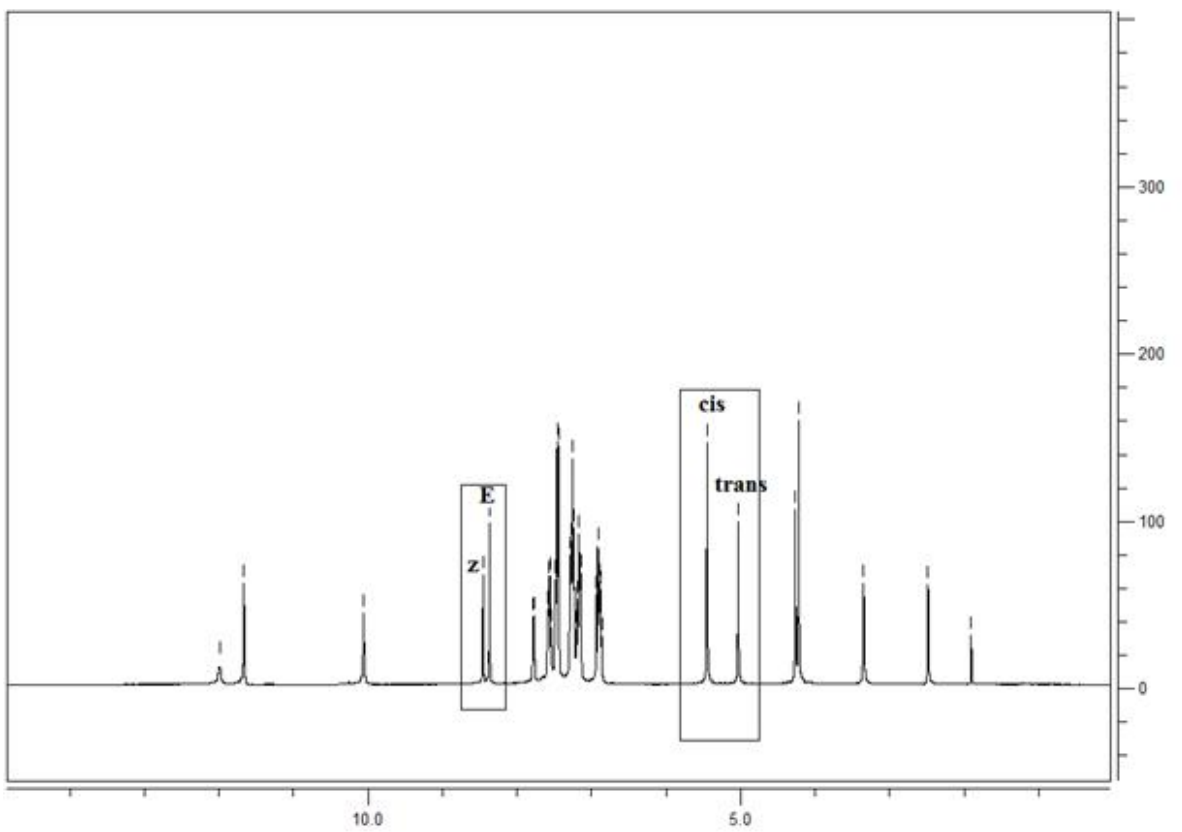

Figure 1. ${ }^{1} \mathrm{H}-\mathrm{NMR}$ spectra of compound $\mathbf{5 a}$. 
When ${ }^{1} \mathrm{H}-\mathrm{NMR}$ spectra of the compounds have been compared, it has been seen that some of the protons have 2 sets of signal at different ppm. This is because of the compounds, which have arylene-hydrazide structure, exist as $E / Z$ geometrical isomer from $\mathrm{C}=\mathrm{N}$ double bond and cis/trans amide conformer at the CO-NH single bond. According to the literature [11, 44, 45], compounds which have $\mathrm{C}=\mathrm{N}$ double bond prefers $E$ geometrical isomer in DMSO- $d_{6}$ and $Z$ isomers can be preferred in less polar solvents. N-CH signals because of cis/trans conformer. The ratio in each case has been calculated by using ${ }^{1} \mathrm{H}-$ NMR data. $E / Z$ and cis/trans geometrical isomers of compounds 5a-f and 6a-d are shown in Scheme 2. The ${ }^{1} \mathrm{H}-\mathrm{NMR}$ spectra of compound $\mathbf{5 a}$ is given as an example in Figure 1.

In the ${ }^{13} \mathrm{C}$ NMR spectrum of 5a-f and 6a-d shows two signals indicated the appearance of $\mathrm{C}=\mathrm{O}$ groups at about $165 \mathrm{ppm}$ and $160 \mathrm{ppm} . \mathrm{N}=\mathrm{CH}$ carbon was shown at about $145 \mathrm{ppm}$. In addition to this, the elemental analysis and mass spectra results of the compounds $\mathbf{5 a - f}$ and $\mathbf{6 a - d}$ show good agreement with the calculated values.

\section{CONCLUSION}

In conclusion, a new series of potentially bioactive benzimidazole derivatives containing salicyl and isatin moieties were synthesized in a short time and good yields by using microwave irradiation technique. These results can be inspired by researchers for the synthesis of new potential bioactive compounds.

\section{REFERENCES}

1. Parikh, K.; Joshi, D. Antibacterial and antifungal screening of newly synthesized benzimidazole-clubbed chalcone derivatives. Med. Chem. Res. 2013, 22, 3688-3697.

2. Abu-Bakr, S.M.; Bassyouni, F.A.; Rehim, M.A. Pharmacological evaluation of benzimidazole derivatives with potential antiviral and antitumor activity. Res. Chem. Intermediat. 2012, 38, 2523-2545.

3. Thakurdesai, P.; Wadodkar, S.G.; Chopade, C.T. Synthesis and anti-inflammatory activity of some benzimidazole-2-carboxylic acids. Pharmacologyonline 2007, 1, 314-329.

4. Shingalapur, R.V.; Hosamani, K.M.; Keri, R.S.; Hugar, M.H. Derivatives of benzimidazole pharmacophore: Synthesis, anticonvulsant, antidiabetic and DNA cleavage studies. Eur. J. Med. Chem. 2010, 45, 1753-1759.

5. Zhang, Y.; Xu, J.; Li, Y.; Yao, H.; Wu, X. Design, synthesis and pharmacological evaluation of novel no-releasing benzimidazole hybrids as potential antihypertensive candidate. Chem. Biol. Drug Des. 2015, 85, 541-548.

6. Menteşe, E.; Karaali, N.; Yılmaz, F.; Ülker, S.; Kahveci, B. Microwave-assisted synthesis and biological evaluation of somebenzimidazole derivatives containing a 1,2,4-triazol ring. Arch. Pharm. Chem. Life Sci. 2013, 346, 556-561.

7. Menteşe, E.; Yilmaz, F.; Karaali, N.; Ülker, S.; Kahveci, B. Rapid synthesis and lipase inhibition activity of some new benzimidazole and perimidine derivatives. Russ. J. Bioorg. Chem. 2014, 40, 336-342.

8. Menteşe, E.; Yilmaz, F.; Baltaş, N.; Bekircan, O.; Kahveci, B. Synthesis and antioxidant activities of some new triheterocyclic compounds containing benzimidazole, thiophene, and 1,2,4-triazole rings. J. Enzyme Inhib. Med. Chem. 2015, 30, 435-441.

9. Menteşe, E.; Bektaş, H.; Ülker, S.; Bekircan, O.; Kahveci, B. Microwave-assisted synthesis of new benzimidazole derivatives with lipase inhibition activity. J. Enzyme Inhib. Med. Chem. 2014, 30, 64-68.

10. Menteşe E.; Ülker S.; Kahveci B. Synthesis and study of alpha-glucosidase inhibitory, antimicrobial and antioxidant activities of some benzimidazole derivatives containing 
triazole, thiadiazole, oxadiazole, and morpholine rings. Chem. Heterocycl. Comp. 2015, 50, 1671-1682.

11. Kahveci, B.; Yılmaz, F.; Menteşe, E.; Özil, M.; Karaoğlu, Ş. A. Microwave-assisted synthesis of some novel benzimidazole derivatives containing imine function and evaluation of their antimicrobial activity. J. Heterocycl. Chem. 2014, 51, 982-990.

12. Kahveci, B.; Menteșe, E.; Özil, M.; Ülker, S.; Ertürk, M. An efficient synthesis of benzimidazoles via a microwave technique and evaluation of their biological activities. Monatsh. Chem. 2013, 144, 993-1001.

13. Usta, A.; Yılmaz, F.; Kapucu, G.; Baltas, N.; Menteşe, E. Synthesis of some new benzimidazole derivatives with their antioxidant activities. Lett. Org. Chem. 2015, 12, 227 232.

14. Karaali, N.; Menteşe, E. Synthesis and study of antitumor activity of some new 2-(4methoxybenzyl)-1h-benzımidazole derivatives bearing triazole, oxadiazole and ethanol moiety. Rev. Roum. Chim. 2016, 61, 187-192.

15. Mentese, E.; Bekircan, O.; Islamoglu, F.; Beris, F.B. Microwave assisted synthesis of some novel 2-(3-chlorobenzyl)-1H-benzimidazole derivatives and determination of their antimicrobial activity and $\mathrm{pK}_{\mathrm{a}}$ values. Rev. Chim. Bucharest 2015, 66, 25-31.

16. Williams, D.R. Metals, ligands, and cancer. Chem. Rev. 1972, 72, 203-213.

17. Campos, A.; Anacona J.R.; Campos-Vallette, M.M. Synthesis and IR study of a zinc(II) complex containing a tetradentate macrocyclic Schiff base ligand: Antifungal properties. Group. Metal Chem. 1999, 22, 283-288.

18. Sari, N.; Arslan, S.; Logoglu, E.; Sakiyan, I. Antibacterial activities of some new amino acid-Schiff bases. G. U. J. Sci. 2003, 16, 283-288.

19. Verma, M.; Pandeya, S.N.; Singh, K.N.; Stabler, J.P. Anticonvulsant activity of Schiff bases of isatin derivatives. Acta Pharm. 2004, 54, 49-56.

20. Cozzi, P.G. Metal-salen Schiff base complexes in catalysis: Practical aspects. Chem. Soc. Rev. 2004, 33, 410-421.

21. Chandra, S.; Sangeetika, J. EPR and electronic spectral studies on copper(II) complexes of some N-O donor ligands. J. Indian Chem. Soc. 2004, 81, 203-206.

22. Kahveci, B.; Menteşe, E.; Akkaya, E.; Yılmaz, F.; Doğan, İ.S.; Özel, A. Synthesis of some novel 1,2,4-triazol-3-one derivatives bearing the salicyl moiety and their anticonvulsant activities. Arch. Pharm. Chem. Life Sci. 2014, 347, 449-455.

23. Pandeya, S.N.; Smitha, S.; Jyoti, M.; Sridhar, S.K. Biological activities of isatin and its derivatives. Acta Pharm. 2005, 55, 27-46.

24. Pandey, V.K.; Dwivedi, A.; Pandey, O.P.; Sengupta, S.K. Organophosphorus derivatives containing isatin-3-hydrazones as chemotherapeutants against fungal pathogens of sugarcane. J. Agric. Food Chem. 2008, 56, 10779-10784.

25. Wang, J.; Tan, H.; Li, Y.; Li, Z.; Guddat, L.W. Chemical synthesis, in vitro acetohydroxyacid synthase (AHAS) inhibition, herbicidal activity, and computational studies of isatin derivatives. J. Agric. Food Chem. 2011, 59, 9892-9900.

26. Menteşe, E.; Yılmaz, F.; İslamoğlu, F.; Kahveci B. Rapid and efficient synthesis of a new series of 2-aryl-5-fluoro-6(4-phenylpiperazin-1-yl)-1H-benzimidazoles using microwave heating. J. Chem. Res. $-S$ 2015, 39, 206-208.

27. Kahveci, B.; Sosan, N.; Mentese, E., Y1lmaz F. Microwave-assisted synthesis of some novel benzimidazole compounds containing oxadiazole moiety. Rev. Roum. Chim. 2013, 58, 511515 .

28. Mentese, E.; Kahveci, B. Microwave-assisted synthesis of some 2-substituted quinazolin$4(3 \mathrm{H})$-one derivatives from iminoester hydrochlorides. Rev. Roum. Chim. 2014, 59, $147-$ 150 . 
29. Mentese, E.; Doğan, İ.S.; Kahveci, B. Green protocol: Solvent- and catalyst-free synthesis of benzimidazole derivatives via microwave technique. Chem. Heterocycl. Comp. 2013, 49, 1136-1140.

30. Özil, M.; Menteşe, E.; Yılmaz, F.; İslamoğlu, F.; Kahveci B. Synthesis of novel triazole compounds containing isatin as potential antibacterial and antifungal agents by microwave and conventional methods. J. Chem. Res. 2011, 35, 268-271.

31. Bekircan, O.; Menteşe, E.; Ülker, S.; Küçük, Ç. Synthesis of some novel 1,2,4-triazol-3-one derivatives bearing the salicyl moiety and their anticonvulsant activities. Arch. Pharm. Chem. Life Sci. 2014, 347, 387-397.

32. Menteşe, E. Efficient microwave assisted synthesis of some new benzimidazoles containing the mebendazole nucleus. J. Chem. Res. 2013, 37, 168-170.

33. Menteşe, E.; Yılmaz, F.; Mutlu, F.; Kahveci, B. Synthesis of new coumarin containing benzimidazole derivatives. J. Chem. Res. $-S$ 2015, 39, 645-648.

34. Menteşe, E.; Bektas, H.; Sokmen, B.B.; Emirik M.; Cakir D.; Kahveci B. Synthesis and molecular docking study of some 5,6-dichloro-2cyclopropyl-1H-benzimidazole derivatives bearing triazole, oxadiazole, and imine functionalities as potentinhibitors of urease. Bioorg. Med. Chem. Lett. 2017, .27, 3014-3018.

35. Yilmaz, F.; Menteşe, E.; Baltas N. Synthesis and antioxidant evaluation of some novel benzimidazole derivatives containing a triazole nucleus. Lett. Drug Dis. Discov. 2017, 14, 201-208.

36. Bekircan, O.; Baltaş, N.; Menteşe, E.; Gultekin E. Synthesis of new fluorine-containing 1,2,4-triazole-5-on derivatives with their anti-urease, anti-xanthine oxidase and antioxidant activities. Rev. Roum. Chim. 2016, 61, 733-746.

37. Menteşe, E.; Karaali, N.; Akyüz, G.; Yilmaz, F.; Ülker, S.; Kahveci, B. Synthesis and evaluation of alpha-glucosidase and pancreatic lipase inhibition by quinazolinonecoumarinhybrids. Chem. Heterocycl. Comp. 2016, 52, 1017-1024.

38. Kahveci, B.;Yilmaz, F.; Menteşe, E.; Ülker, S. Design, synthesis, and biological evaluation of coumarin-triazole hybrid molecules as potential antitumor and pancreatic lipase agents. Arch. Pharm. Chem. Life Sci. 2017, 350, 1-9.

39. Yilmaz, F.; Menteşe, E. Synthesis and characterisation of some coumarin-1,2,4-triazol-3thioether hybrid molecules. J. Chem. Res. 2017, 41, 4-6.

40. Baltaş, N.; Yilmaz, F.; Menteşe, E. Synthesis, antioxidant, xanthine oxidase and urease inhibitory activities of some chlorine containing benzimidazoles. Hacettepe J. Biol. Chem. 2016, 44, 293-305.

41. Emirik, M.; Karaoğlu, K.; Serbest, K.; Menteşe, E.; Yılmaz, İ. Ferrocenyl-substituted dinuclear $\mathrm{Cu}(\mathrm{II})$ complex: Synthesis, spectroscopy, electrochemistry, DFT calculations and catecholase activity. J. Mol. Struct. 2016, 1106, 331-342.

42. Menteşe, E.; Kahveci, B. Efficient synthesis of benzimidazoles containing 1,2,4-triazol-3one nucleus using microwave technique. Chem. Heterocycl. Comp. 2016, 52, 948-951.

43. Yilmaz, F.; Menteşe, E.; Karaali, N.; Kahveci, B. Microwave-assisted synthesis of some 5(6)-nitro-1H-benzimidazoles and their hydrazide derivatıves. Bull. Chem. Soc. Ethiop. 2013, 27, 265-271.

44. Galic, N.; Peric, B.; Kojic-Prodic, B.; Cimerman, Z. Structural and spectroscopic characteristics of aroylhydrazones derived from nicotinic acid hydrazide. J. Mol. Stuc. 2001, $559,187-194$.

45. Bektaş, H.; Demirbaş, A.; Demirbaş, N.; Karaoğlu, Ş.A. Synthesis of some new biheterocyclic triazole derivatives and evaluation of their antimicrobial activity. Turk. $J$. Chem. 2010, 34, 165-180. 